\title{
Development and validation of bivariate UV-visible spectroscopic method for simultaneous estimation of curcumin and piperine in their combined nanoparticulate system
}

\author{
Srinivas Bhairy ${ }^{1}$, Ashraf Shaikh², Vivek Nalawade ${ }^{2}$, Rajashree Hirlekar ${ }^{1 *}$ \\ ${ }^{1}$ Department of Pharmaceutics, Vivekanand Education Societys College of Pharmacy, Mumbai, India. \\ ${ }^{2}$ Department of Quality Assurance, Vivekanand Education Societys College of Pharmacy, Mumbai, India.
}

\begin{tabular}{l}
\hline ARTICLE INFO \\
\hline Received on: 06/06/2020 \\
Accepted on: $17 / 12 / 2020$ \\
Available online: $05 / 05 / 2021$ \\
\\
\hline Key words: \\
Curcumin, piperine, \\
nanoparticulate system, \\
spectrophotometric analysis, \\
simultaneous equation \\
method.
\end{tabular}

\begin{abstract}
The purpose of the research was to develop ultraviolet (UV) spectroscopic methods to simultaneously estimate curcumin (CUR) and piperine (PIP). This method involves interpreting simultaneous equations using UV visible spectrophotometer with $1 \mathrm{~cm}$ matched quartz cells and methanol as a solvent based on measuring absorbance at two wavelengths of 423 and $342 \mathrm{~nm}$. The method developed obeyed the law of Beer-Lambert in the concentration range of $1-7 \mu \mathrm{g} / \mathrm{ml}$, with the correlation coefficient for CUR and PIP at their respective maximum wavelengths being 0.9990 and 0.9988 , respectively. At an iso-absorptive point, CUR and PIP in the concentration range of 1-7 $\mu \mathrm{g} / \mathrm{ml}$ showed a correlation coefficient of 0.9995 and 0.9988 , respectively. Various validation parameters, such as precision (intraday and interday studies), limit of detection, and limit of quantitation, have been tested and found to be within the limit. The results of the method have been statistically validated. For the simultaneous estimation of CUR and PIP, a novel, simple, sensitive, fast, accurate, and economical spectrophotometric method has been developed. The method can be used to estimate the amount of CUR and PIP in a nanoparticulate system containing CUR and PIP.
\end{abstract}

\section{INTRODUCTION}

Curcumin (CUR) is a crystalline orange-yellow phytochemical that is isolated from Curcuma longa (Zingiberaceae) along with other two de-methoxy compounds which are desmethoxycurcumin and bisdemethoxycurcumin, and are classified as a functional food. Structurally, CUR is 1, 7- bis (4-hydroxy-3-methoxyphenyl)-1,6-heptadiene-3,5-dione (Figure 1A) (Suchitra and Rajashree, 2016). Since its diverse molecular targets, it exhibits a wide variety of pharmacological activities including anti-cancer activity. CUR also possesses anti-inflammatory, chemo-sensitizing, radio-sensitizing, wound healing, antimicrobial, antiviral, antifungal, immunomodulatory, and antioxidant activities. CUR is relatively healthy and can be

"Corresponding Author

Rajashree Hirlekar, Department of Pharmaceutics, Vivekanand Education Societys College of Pharmacy, Mumbai, India. E-mail: rajashree.hirlekar@ves.ac.in strongly tolerated well at high doses and has also been declared generally recognized as safe (GRAS) by the United States Food and Drug Administration (USFDA) (Moorthi and Kathiresan, 2012; Moorthi and Kathiresan, 2013; Moorthi et al., 2011, 2012; Sharma et al., 2005). PIP is a major alkaloid isolated from Piper nigrum L. (Piperaceae). Structurally, PIP is 1-[(2E,4E)-5-(1,3Benzodioxol-5-yl)-1-oxo-2,4-pentadieneyl] piperidine (Figure1B) (Maryadele, 2001). The most interesting point is that PIP increases the bioavailability of a number of therapeutic drugs, as well as phytochemicals, including CUR (Vipul et al., 2013). PIP has several reported pharmacological activities like central nervous system depressant, antipyretic, analgesic, hepatoprotective, antioxidant, and anti-inflammatory (Hamrapurkar et al., 2011). It is also listed by the USFDA as a GRAS molecule (Nurul et al., 2010).

The simultaneous estimate of CUR and PIP in their combined dosage form is not standard in any pharmacopoeia; therefore, no standard method of estimating CUR and PIP in their combined dosage forms is available (Kaushik et al., 2012). A literature survey reported that several methods are available for the estimation of CUR and PIP simultaneously in different formulations 

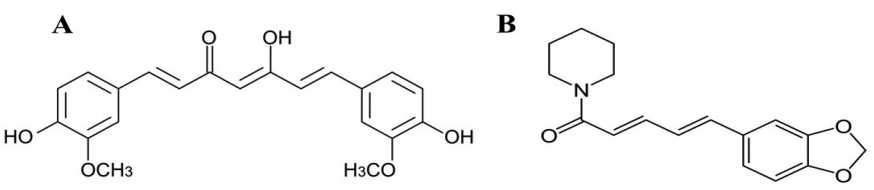

Figure 1. Structure of (A) CUR and (B) PIP.

and in plasma. Namely, reverse phase high performance liquid chromatography (RP-HPLC) with photodiode array (PDA) detector (Moorthi et al., 2013), HPLC with electrospray tandem identification of the mass spectrometer in strong ionization mode (Xiu-Mei et al., 2012), RP-HPLC system with a fluorescence detector (Shaikh et al., 2009), RP-HPLC method using diode array detection system (Nagappan et al., 2009), RP-HPLC with ultraviolet (UV)/Visible Detector (Chahar and Mashru, 2016), ultra-fast liquid chromatography using a PDA (Shanmugam et al., 2014), and HPLC system UV-Visible detector(Prerana et al., 2009). Furthermore, it was found that only one method was developed by using UV Spectrophotometer, but it was for a mixture of CUR, PIP, and quercetin (Aneja et al., 2012). A UV-Visible spectroscopic approach was developed to estimate the dissolution profile of CUR, PIP, and their accurate measurement in formulated 0.5 Sodium lauryl sulfate (SLS) dissolution medium in $\mathrm{pH} 6.0$ sodium phosphate buffer (Murti et al., 2019). The reported methods were not cost-effective due to the use of highly sophisticated instruments and detectors and costly solvents, such as tetrahydrofuran, trifluoro acetic acid, and some methods, were found to be less sensitive. The reported UV-Visible spectroscopic method was highlighting the estimation of CUR and PIP in the dissolution medium of the formulation. So in this research work, a quick, simplified, accurate, and sensitive simultaneous estimation approach (Vierordt's and Q absorbance methods) was developed by using low-cost solvent methanol by using UV-Visible spectrophotometer which was highly sensitive to detect lower concentration. The developed approach was used for estimating the CUR and PIP in CUR+PIP dual drugloaded nanostructured lipid carriers (NLCs) in which methanol was used for extraction of CUR and PIP. The use of methanol as an extracting solvent makes it more compatible with the developed method.

\section{METHODS AND MATERIALS}

\section{Materials}

CUR of purity $99.50 \%$ was obtained from VAV Life Science (Mumbai, India) as a gift sample. PIP of purity $97.00 \%$ was bought from Sigma-Aldrich (Mumbai, India). Analytical reagent grade methanol of purity $99.80 \%$ was bought from Rankem chemicals (Mumbai, India). Glyceryl distearate (Precirol ATO5), medium chain triglycerides (Labrafac Lipophile WL1349), and Stearoyl polyoxyl-32 glycerides (Gelucire 50/13) were gifted by Gattefosse (Mumbai, India). Polysorbate 80 (Tween 80) was gifted by BASF (Mumbai, India).

\section{Instrumentation}

For spectra and absorbance imaging and calculation, a double beam UV-visible spectrophotometer (SHIMADZU UV1800) (Mumbai, India) consisting of two matched quartz cells with $1 \mathrm{~cm}$ light direction and fitted with UV probe program (version 2.3) was used. In this project, an electronic analytical weighing balance (Shimadzu AU 220) (Mumbai, India) and a sonicator (Sonica, model 2200 MH) (Mumbai, India) were used.

\section{Method development}

Selection of the solvent

Various solvent mixtures were studied. The parameters used for the selection of solvent were the ease of processing the standards and samples, solubility, and stability of actives, cost, and applicability for the method. Both the actives showed good solubility profile in methanol which is inexpensive and thus it was chosen for the study.

\section{Preparation of Standard stock solutions}

Stock solutions containing standard CUR and PIP were independently prepared by dissolving $10 \mathrm{mg}$ of CUR and $10 \mathrm{mg}$ of PIP in $10 \mathrm{ml}$ of methanol, respectively; the prepared solutions were sonicated for $10 \mathrm{~min}$ and the final volume of both solutions was rendered up to $100 \mathrm{ml}$ with the same solvent to obtain stock solutions comprising $100 \mu \mathrm{g} / \mathrm{ml}$ of CUR and PIP each.

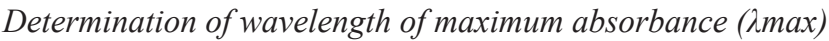 and Iso-absorptive Point}

The standard CUR and PIP stock solutions were diluted individually with methanol to provide a solution comprising $10 \mu \mathrm{g} / \mathrm{ml} \mathrm{CUR}$ and $10 \mu \mathrm{g} / \mathrm{ml}$ PIP. Approximately $3.0 \mathrm{ml}$ was collected and tested with the UV spectrophotometer from 200 to $800 \mathrm{~nm}$. The wavelengths were selected in such a way that at each wavelength the absorptivity difference between the two actives was as large as possible. Considering this, the $\lambda_{\text {max }}$ of both actives were selected for the method.

\section{Preparation of standard calibration curve of CUR and PIP}

In both CUR and PIP, a calibration curve was plotted at a concentration range of $1-30 \mu \mathrm{g} / \mathrm{ml}$. Accurately, measured working stock solutions $(1,2,3,4,5,6$, and $7 \mu \mathrm{g} / \mathrm{ml})$ for both CUR and PIP were prepared using methanol as a solvent in two different series of $10 \mathrm{ml}$ volumetric flask. All the solutions were screened for absorbance at their respective $\lambda_{\max }$ and iso-absorptive point. The calibration curves were developed by plotting the concentration against absorbance where every reading was mean of three determinations.

\section{Sample preparation method}

The sample solution in the ration of 2:1 (CUR:PIP) was prepared from standard stock solutions. Sample solution absorbance was measured simultaneously at 423, $342 \mathrm{~nm}$, and also at $368.5 \mathrm{~nm}$ at its iso-absorptive point.

\section{Preparation of CUR+PIP NLCs formulation}

It was prepared by a modified hot melt emulsification/ ultrasonication technique using lipids and surfactants at Vivekanand Education Society's College of Pharmacy (Chembur, Mumbai). The formulation contains $3 \% \mathrm{w} / \mathrm{v}$ of glyceryl distearate (Precirol ATO5) as solid lipid, 2\% $w / v$ medium chain triglycerides (Labrafac Lipophile WL1349) as liquid lipid, and combination of $1.25 \% \mathrm{w} / \mathrm{v}$ of solid surfactant stearoyl polyoxyl-32 glycerides (Gelucire 50/13) with $1.25 \%$ w/v liquid surfactant Polysorbate 80 (Tween 80). The prepared nanoparticulate formulation contained $0.08 \% w / v$ of CUR and $0.04 \% w / v$ of PIP. 


\section{Method I (Vierordt's simultaneous equation method)}

The research is focused on the absorption of actives CUR and PIP at their wavelength maxima in this simultaneous equation system (Mohit et al., 2010). Two chosen wavelengths for the simultaneous calculations are 423 and $342 \mathrm{~nm}$. The absorptivity measurement values for CUR are $1.377\left(\mathrm{ax}_{1}\right), 0.270$ $\left(\mathrm{ax}_{2}\right)$, and for PIP are $0.004\left(\mathrm{ay}_{1}\right), 1.257\left(\mathrm{ay}_{2}\right)$ at 423 and $342 \mathrm{~nm}$, respectively. These measurements are a mean of six estimations. This determines the concentration of actives and the absorbances at these wavelengths were substituted in equations (1) and (2).

$$
\begin{aligned}
& C_{x}=\frac{A_{2} * a y_{1}-A_{1}^{*} a y_{2}}{a x_{2} * a y_{1}-a x_{1}^{*} a y_{2}} \\
& C_{y}=\frac{A_{1} * a x_{1}-A_{2}^{*} a x_{1}}{a x_{2} * a y_{1}-a x_{1}^{*} a y_{2}}
\end{aligned}
$$

where,

$\mathrm{C}_{\mathrm{x}}$ and $\mathrm{C}_{\mathrm{y}}$ are concentrations of CUR and PIP in $\mu \mathrm{g} / \mathrm{ml}$ in sample solution, respectively.

$A_{1}$ and $A_{2}$ are the absorbance of sample solutions at $423 \mathrm{~nm}$ and $342 \mathrm{~nm}$, respectively.

$\mathrm{a}_{\mathrm{x} 1}$ is the absorbance of CUR at $423 \mathrm{~nm}$.

$\mathrm{a}_{\mathrm{x} 2}$ is the absorbance of CUR at $342 \mathrm{~nm}$.

$\mathrm{a}_{\mathrm{y} 1}$ is the absorbance of PIP at $423 \mathrm{~nm}$.

$\mathrm{a}_{\mathrm{y} 2}$ is the absorbance of PIP at $342 \mathrm{~nm}$.

By substituting the values of $\mathrm{A}_{1}$ and $\mathrm{A}_{2}$, the $\mathrm{C}_{\mathrm{x}}$ and $\mathrm{C}_{\mathrm{y}}$ can be calculated by solving equations (1) and (2).

\section{Method II (absorbance ratio or Q-analysis method)}

In the absorption ratio approach (Tushar et al., 2014), absorbances of both the actives are measured at two chosen wavelengths among which $\lambda_{1}$ is the $\lambda_{\text {max }}$ of either drug among both drugs and $\lambda_{2}$ is the wavelength of iso-absorptive point of both drugs. Here, $\lambda_{1}$ is selected as $423 \mathrm{~nm}$ and from the overlain spectra wavelength $\left(\lambda_{2}\right) 368.5 \mathrm{~nm}$ (iso-absorption point) were selected for the formation of q absorbance equation [Eqs. (3) and (4)]. The absorbances at 423 and $368.5 \mathrm{~nm}$ for CUR were obtained and similarly for PIP absorbances are measured at 423 and $368.5 \mathrm{~nm}$. The concentration of the individual actives was determined by using the following equations:

$$
\begin{aligned}
C_{x} & =\frac{Q m-Q y}{Q x-Q y} * \frac{A_{1}}{a x} \\
C_{y} & =\frac{Q m-Q y}{Q y-Q x} * \frac{A_{1}}{a y}
\end{aligned}
$$

Where,

$\mathrm{C}_{\mathrm{x}}$ and $\mathrm{C}_{\mathrm{y}}$ are concentrations of CUR and PIP, respectively, $\mathrm{Q}_{\mathrm{x}}=$ the ratio of absorbances of CUR at 423 and $368.5 \mathrm{~nm}$.

$\mathrm{Q}_{\mathrm{y}}=$ the ratio of absorbances of PIP at 423 and $368.5 \mathrm{~nm}$.

$\mathrm{Q}_{\mathrm{m}}=$ the ratio of absorbance of mixture at 423 and $368.5 \mathrm{~nm}$.

$\mathrm{A}_{1}=$ the absorbance of mixture at iso-absorptive point.

$\mathrm{a}_{\mathrm{x}}=$ the absorbance value of CUR at iso-absorptive point.

$\mathrm{a}_{\mathrm{y}}=$ the absorbance value of PIP at iso-absorptive point.

\section{Method validation}

The methods mentioned have been validated using International Conference on Harmonization (ICH) parameters for the assay of the two active components of the mixture (ICH, 2005).

\section{Linearity}

Linearity was tested by using prepared standard solutions of actives at varying levels of concentration. Calibration curves were constructed using the standard solutions of $1-7 \mu \mathrm{g} / \mathrm{ml}$ and linear regression analysis was conducted.

\section{Precision}

The intraday and interday precision of the developed method was evaluated by determining the related response three times on the same day, and three separate concentrations $(3: 3,5: 5$, $7: 7 \mu \mathrm{g} / \mathrm{ml}$ ) on two different days.

\section{Accuracy (\% recovery study)}

The accuracy of the developed analytical method shows the closeness of agreement between the value recognized either as a traditional truth value or as an agreed reference value and the observed value. The recovery tests were carried out in triplicate by spiking samples previously studied with three separate concentrations of standards.

\section{Limit of detection (LOD) and Limit of Quantitation (LOQ)}

The LOD and the LOQ of the actives were obtained by calculating the signal-to-noise ratio $(\mathrm{S} / \mathrm{N})$ i.e., 3.3 for $\mathrm{LOD}$ and 10 for LOQ using the following equation as defined by the $\mathrm{ICH}$ guidelines.

$$
\begin{aligned}
& \mathrm{LOD}=\frac{3.3 \sigma}{S} \\
& \mathrm{LOQ}=\frac{10 \sigma}{S}
\end{aligned}
$$

Where, $\sigma=$ standard deviation response and $\mathrm{S}=$ slope of the calibration curve.

\section{Application of validated method for assay of CUR+PIP in NLCs formulation}

In a volumetric flask, $1 \mathrm{ml}$ formulation of CUR+PIP NLCs (equivalent to $0.80 \mathrm{mg}$ of CUR and $0.40 \mathrm{mg}$ of PIP) was combined with methanol up to $10 \mathrm{ml}$. This was subjected to 60 min of bath sonication for complete CUR and PIP extraction. The sonicated solution was mixed and was purified using a $0.45 \mu$ Nylon syringe filter to get an $80 \mu \mathrm{g} / \mathrm{ml}$ of CUR and $40 \mu \mathrm{g} / \mathrm{ml}$ of PIP solution. The solution was further appropriately diluted and analysed UV-Visible spectrophotometer utilizing the simultaneous equations for the estimation of actives.

\section{RESULTS AND DISCUSSION}

\section{Method development}

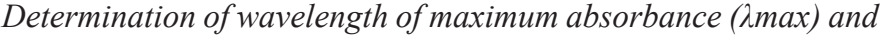
Iso-absorptive Point

CUR exhibits maximum absorption at a wavelength $\left(\lambda_{\max }\right) 423 \mathrm{~nm}$, while PIP exhibits maximum absorption at a wavelength $\left(\lambda_{\max }\right) 342 \mathrm{~nm}$ and from overlain spectra, it is evident that the iso-absorptive point is as $368.5 \mathrm{~nm}$. 


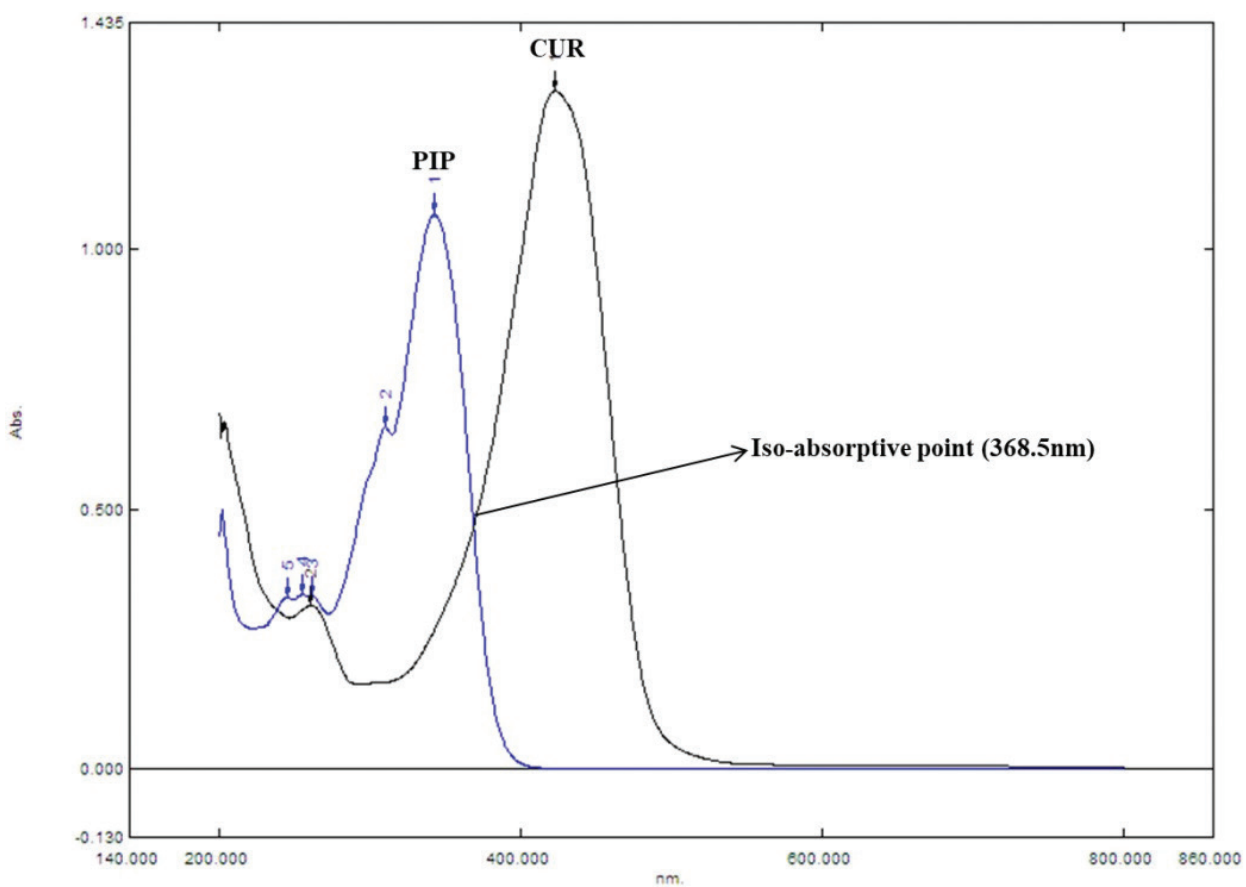

Figure 2. UV overlay spectra of CUR and PIP.

Standard calibration curve of CUR and PIP
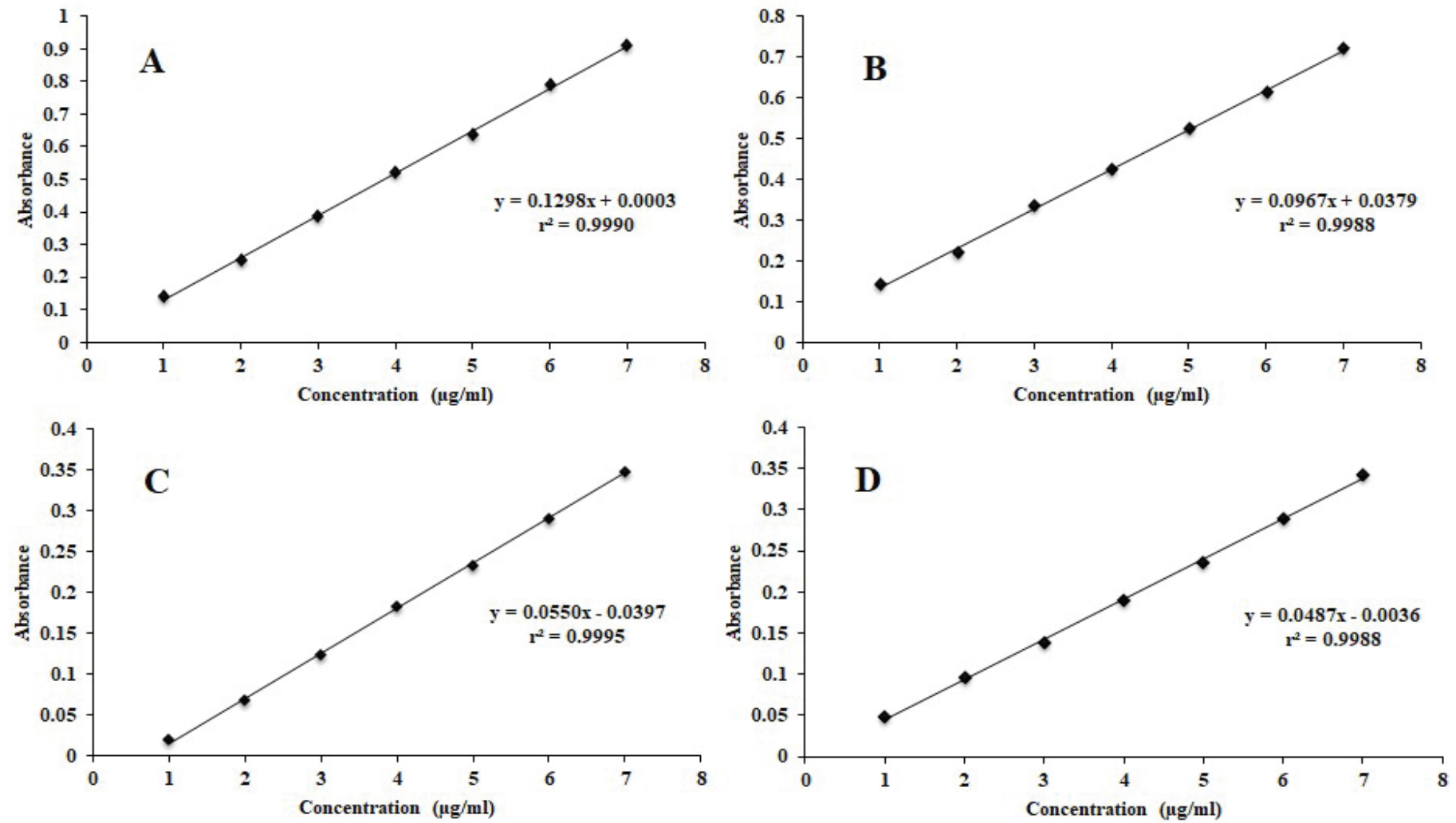

Figure 3. Calibration curve of (A) CUR in methanol at wavelength $\left(\lambda_{\max }\right) 423 \mathrm{~nm}$, (B) PIP in methanol in methanol at wavelength $\left(\lambda_{\max }\right) 342 \mathrm{~nm}$, (C) CUR in methanol at iso-absorptive point $368.5 \mathrm{~nm}$, (D) PIP in methanol at iso-absorptive point $368.5 \mathrm{~nm}$. 


\section{Method validation}

Linearity

The regression coefficients are reported in Table 1.

Table 1. Linear regression analysis of calibration curves of CUR and PIP with both the methods.

\begin{tabular}{ccccc}
\hline \multirow{2}{*}{$\begin{array}{c}c \\
\text { Concentration }\end{array}$} & \multicolumn{2}{c}{ Method I } & \multicolumn{2}{c}{ Method II } \\
\cline { 2 - 5 }$(\mu \mathbf{g} / \mathbf{m l})$ & $\begin{array}{c}\text { Absorbance } \\
\text { at } \mathbf{4 2 3} \mathbf{~ n m}\end{array}$ & $\begin{array}{c}\text { Absorbance } \\
\text { at 342 } \mathbf{~ n m}\end{array}$ & $\begin{array}{c}\text { Absorbance at } \\
\text { Iso-absorptive } \\
\text { point 368.5 } \mathbf{~ m m}\end{array}$ & $\begin{array}{c}\text { Absorbance at } \\
\text { Iso-absorptive } \\
\text { point 368.5 nm }\end{array}$ \\
\cline { 2 - 5 } 1 & 0.140 & 0.142 & 0.019 & 0.049 \\
2 & 0.253 & 0.219 & 0.068 & 0.095 \\
3 & 0.386 & 0.334 & 0.123 & 0.139 \\
4 & 0.523 & 0.422 & 0.182 & 0.189 \\
5 & 0.635 & 0.525 & 0.232 & 0.235 \\
6 & 0.788 & 0.612 & 0.290 & 0.289 \\
7 & 0.912 & 0.719 & 0.348 & 0.342 \\
$r^{2}$ & 0.9990 & 0.9988 & 0.9995 & 0.9988 \\
Intercept & 0.0003 & 0.0379 & 0.0397 & 0.0036 \\
\hline
\end{tabular}

\section{Precision}

The result was reported in terms of \%relative standard deviation (RSD).

\section{$L O D$ and $L O Q$}

Table 4. Results of LOD and LOQ.

\begin{tabular}{lcccc}
\hline \multirow{2}{*}{ Components } & \multicolumn{2}{c}{ Method I } & \multicolumn{2}{c}{ Method II } \\
\cline { 2 - 5 } & LOD $(\mu \mathrm{g} / \mathrm{ml})$ & LOQ $(\mu \mathrm{g} / \mathrm{ml})$ & LOD $(\mu \mathrm{g} / \mathrm{ml})$ & LOQ $(\mu \mathrm{g} / \mathrm{ml})$ \\
\hline CUR & 0.0926 & 0.2807 & 0.0683 & 0.2071 \\
PIP & 0.1027 & 0.3112 & 0.1024 & 0.3103 \\
\hline
\end{tabular}

\section{Assay of CUR+PIP in NLCs formulation}

Table 5. Assay results of CUR +PIP NLCs formulation

\begin{tabular}{lcccc}
\hline \multirow{2}{*}{ Parameters } & \multicolumn{4}{c}{ Results } \\
\cline { 2 - 5 } & \multicolumn{2}{c}{ Method I } & \multicolumn{2}{c}{ Method II } \\
\cline { 2 - 5 } & CUR & PIP & CUR & PIP \\
\hline Amount of drug added (mg) & 0.80 & 0.40 & 0.80 & 0.40 \\
Amount of drug recovered (mg) & 0.77 & 0.39 & 0.78 & 0.39 \\
Assay (\%) & 96.80 & 97.55 & 97.50 & 97.55 \\
\hline
\end{tabular}

The research was aimed at developing and validating a spectroscopic approach for estimating CUR and PIP actives in a nanoparticulate formulation system. The aim of the analytical method validation is to show the suitability and reliability of an analytical method for its intended function. Although many chromatographic methods are found in many publications for

Table 2. Data of precision and accuracy study for CUR and PIP for method I.

\begin{tabular}{|c|c|c|c|c|c|c|c|c|c|}
\hline \multicolumn{5}{|c|}{ Intraday precision } & \multicolumn{5}{|c|}{ Interday precision } \\
\hline \multirow{2}{*}{$\frac{\text { Amount taken (mg) }}{\text { CUR:PIP }}$} & \multicolumn{2}{|c|}{ Amount found (mg) } & \multicolumn{2}{|c|}{ \% Recovery } & \multirow{2}{*}{$\begin{array}{c}\text { Amount taken (mg) } \\
\text { CUR:PIP }\end{array}$} & \multicolumn{2}{|c|}{ Amount found (mg) } & \multicolumn{2}{|c|}{ \% Recovery } \\
\hline & CUR & PIP & CUR & PIP & & CUR & PIP & CUR & PIP \\
\hline $3: 3$ & 2.9676 & 3.0569 & 98.92 & 101.90 & $3: 3$ & 2.9676 & 3.062 & 98.92 & 102.07 \\
\hline $3: 3$ & 2.9908 & 3.0776 & 99.69 & 102.59 & $3: 3$ & 2.9638 & 3.0776 & 98.79 & 102.59 \\
\hline $3: 3$ & 2.9792 & 3.0465 & 99.31 & 101.55 & $3: 3$ & 2.9599 & 3.0724 & 98.66 & 102.41 \\
\hline $5: 5$ & 4.8975 & 5.0372 & 97.95 & 100.74 & $5: 5$ & 4.9014 & 5.0527 & 98.03 & 101.05 \\
\hline $5: 5$ & 4.9129 & 5.0527 & 98.26 & 101.05 & $5: 5$ & 4.8937 & 5.0527 & 97.87 & 101.05 \\
\hline $5: 5$ & 4.8898 & 5.0476 & 97.80 & 100.95 & $5: 5$ & 4.8821 & 5.0321 & 97.64 & 100.64 \\
\hline $7: 7$ & 7.0085 & 7.0434 & 100.12 & 100.62 & $7: 7$ & 7.0123 & 7.0434 & 100.18 & 100.62 \\
\hline $7: 7$ & 7.0354 & 7.0538 & 100.51 & 100.77 & $7: 7$ & 7.0277 & 7.0589 & 100.40 & 100.84 \\
\hline $7: 7$ & 7.0354 & 7.0538 & 100.51 & 100.77 & $7: 7$ & 7.0431 & 7.0279 & 100.62 & 100.40 \\
\hline
\end{tabular}

Table 3. Data of precision and accuracy study for CUR and PIP for method II.

\begin{tabular}{|c|c|c|c|c|c|c|c|c|c|}
\hline \multicolumn{5}{|c|}{ Intraday precision } & \multicolumn{5}{|c|}{ Interday precision } \\
\hline \multirow{2}{*}{$\begin{array}{c}\text { Amount taken (mg) } \\
\text { CUR:PIP }\end{array}$} & \multicolumn{2}{|c|}{ Amount found (mg) } & \multicolumn{2}{|c|}{ \% Recovery } & \multirow{2}{*}{$\begin{array}{c}\text { Amount taken (mg) } \\
\text { CUR:PIP }\end{array}$} & \multicolumn{2}{|c|}{ Amount found (mg) } & \multicolumn{2}{|c|}{ \% Recovery } \\
\hline & CUR & PIP & CUR & PIP & & CUR & PIP & CUR & PIP \\
\hline $3: 3$ & 3.0517 & 3.0375 & 101.72 & 101.25 & $3: 3$ & 3.0315 & 2.9726 & 101.05 & 99.09 \\
\hline $3: 3$ & 3.0897 & 2.9947 & 102.99 & 99.82 & $3: 3$ & 2.9900 & 2.9622 & 99.67 & 98.74 \\
\hline $3: 3$ & 3.0687 & 3.0275 & 102.29 & 100.92 & $3: 3$ & 3.0313 & 2.9943 & 101.04 & 99.81 \\
\hline $5: 5$ & 5.0442 & 5.0048 & 100.88 & 100.10 & $5: 5$ & 4.9477 & 5.0581 & 98.95 & 101.16 \\
\hline $5: 5$ & 5.0859 & 4.9936 & 101.72 & 99.87 & $5: 5$ & 4.9689 & 5.0036 & 99.38 & 100.07 \\
\hline $5: 5$ & 5.0483 & 4.9821 & 100.97 & 99.64 & $5: 5$ & 4.9272 & 5.0257 & 98.54 & 100.51 \\
\hline $7: 7$ & 7.0448 & 6.9267 & 100.64 & 98.95 & $7: 7$ & 7.0318 & 6.9357 & 100.45 & 99.08 \\
\hline $7: 7$ & 7.0068 & 6.9804 & 100.10 & 99.72 & $7: 7$ & 6.9697 & 6.9038 & 99.57 & 98.63 \\
\hline $7: 7$ & 7.0656 & 6.9265 & 100.94 & 98.95 & $7: 7$ & 6.9277 & 6.9585 & 98.97 & 99.41 \\
\hline
\end{tabular}


simultaneous estimation of CUR and PIP in various formulation systems. However, the reported approaches were having some drawbacks such as costly solvents, extensive sample preparation, which may involve multiple clean up steps, followed by extraction procedures and expensive analytical instruments which increases the sample analysis cost (Murti et al., 2019).

The CUR and PIP overlay spectra showed $\lambda_{\max }$ at 423 $\mathrm{nm}$ and $342 \mathrm{~nm}$, respectively, which are quite different from each other. In addition, an iso-absorptive value at $368.5 \mathrm{~nm}$ was found (Figure 2). Standard calibration curves for CUR and PIP were linear with correlation coefficients $\left(r^{2}\right)$ values of 0.9990 and 0.9988 , respectively, at all the selected wavelengths. Standard calibration curves for CUR and PIP were linear with correlation coefficients $\left(r^{2}\right)$ values of 0.9995 and 0.9988 , respectively, at all the iso-aborptive point (Figure 3). The procedure was replicated on the same day and it was observed that the $\%$ RSD was $<0.4 \%$ for CUR and $<0.59$ for PIP, and $<1.4 \%$ at iso-absorptive point, similarly the procedure was replicated on separate days and the $\%$ RSD was found to be $<0.39 \%$ for CUR and $<0.74 \%$ for PIP and $<1.5 \%$ at iso-absorptive point (Table 2 and 3 ). The proposed method showed good accuracy by achieving a good percent recovery in the standard addition method. It ranged between $100.034 \%$ and $101.328 \%$ for CUR and $100.665 \%$ and $102.247 \%$ for PIP. The LOD was observed to be $0.092 \mu \mathrm{g} / \mathrm{ml}$ for CUR and $0.102 \mu \mathrm{g} / \mathrm{ml}$ for PIP. LOQ was observed to be $0.280 \mu \mathrm{g} / \mathrm{ml}$ and $0.311 \mu \mathrm{g} / \mathrm{ml}$ for CUR and PIP, respectively. The LOD of CUR and $\mathrm{PIP}$ at iso-absorptive point $(368.5 \mathrm{~nm})$ was observed to be 0.0683 times and $0.1024 \mu \mathrm{g} / \mathrm{ml}$, respectively. The LOQ of CUR and PIP at iso-absorptive point $(368.5 \mathrm{~nm})$ was observed to be 0.207 and $0.310 \mu \mathrm{g} / \mathrm{ml}$, respectively (Table 4). The developed method was successfully implemented for the assay of CUR+PIP in NLCs formulation. Assay of CUR and PIP was found to be $96.80 \%$ and $97.55 \%$. The extracting solvent was methanol which helped in the complete extraction of CUR and PIP from the formulation. The results are summarized in Table 5. This proposed spectroscopic method confirms linearity, accuracy, and precision of the method. Although spectroscopic methods are not a selective option by analysts, the method is still helpful for the simultaneous estimation of two or more actives in various formulation systems. Despite the selectivity problem, the spectrophotometric method offers simplicity, rapidity, and reliability (Murti et al., 2019).

\section{CONCLUSION}

For the simultaneous estimation of CUR and PIP actives in the nanoparticulate formulation, the UV spectrophotometric Vierordt's simultaneous equation (method I) and Q-absorption ratio (method II) were developed and validated. The suggested methods are simple, rapid, and validated in terms of linearity, accuracy, and precision. The methods can be effectively utilized for the routine estimation of actives CUR and PIP in pure and combined formulated systems.

\section{LIST OF ABBREVIATIONS}

UV - ultraviolet, CUR - Curcumin, PIP - Piperine, nm - Nanometer, $\mathrm{cm}$ - Centimeter, $\mu \mathrm{g} / \mathrm{ml}$ - Microgram per milliliter, LOD - Limit of detection, LOQ - Limit of quantitation, US FDA - United States Food and Drug Administration, GRAS - Generally recognized as safe, RP-HPLC - Reverse Phase high performance liquid chromatography, PDA - Photodiode array, SLS - Sodium lauryl sulfate, NLCs - Nanostructured lipid carriers, ICH International Conference on Harmonization, RSD - Relative standard deviation.

\section{ACKNOWLEDGEMENT}

The authors are thankful to the All India Council for Technical Education, New Delhi, for supporting this work (Ref. no: 8-159/RIFD/RPS/Policy-4/2013-14). The authors are thankful to VAV life sciences for providing gift sample of CUR. They are also thankful to Gattefosse, India, for providing the lipid samples for the development of the nanoparticulate system of CUR and PIP.

\section{AUTHOR CONTRIBUTIONS}

All authors made substantial contributions to conception and design, acquisition of data, or analysis and interpretation of data; took part in drafting the article or revising it critically for important intellectual content; agreed to submit to the current journal; gave final approval of the version to be published; and agree to be accountable for all aspects of the work. All the authors are eligible to be an author as per the international committee of medical journal editors (ICMJE) requirements/guidelines.

\section{CONFLICTS OF INTEREST}

The authors report no financial or any other conflicts of interest in this work.

\section{ETHICAL APPROVALS}

This study does not involve experiments on animals or human subjects.

\section{PUBLISHER'S NOTE}

This journal remains neutral with regard to jurisdictional claims in published institutional affiliation.

\section{REFERENCES}

Aneja G, Dave U, Vadodaria K. Simultaneous estimation of piperine, quercetin, and curcumin in a mixture using U.V-visible spectrophotometer and method validation, Int J Ther Appl, 2012; 8:14-7.

Chahar MY, Mashru R. Development and validation of stability indicating RP-HPLC method for simultaneous estimation of curcumin and piperine in bulk mixture. World J Pharm Res, 2016; 5:1262-76.

Hamrapurkar PD, Kavita J, Sandip Z. Quantitative estimation of piperine in Piper nigrum and piper longum using high performance thin layer chromatography. J App Pharm Sci, 2011; 01:117-120.

ICH. Validation of analytical procedures: text and methodology. In International Conference on Harmonization (ICH), Q2(R1), IFPMA Geneva, Switzerland, 2005.

Kaushik GK, Prakash MP, Hiral RT, Shital DF. Method development and validation of rifampicine and piperine in their combined dosage form. Int Bull Drug Res, 2012, 1:71-80.

Maryadele JO. The Mercek index: an encyclopedia of chemicals, drugs and biological. Whitehouse Station, NJ: The Royal Society of Chemistry.

Mohit R, Abhinav A, Ashish KJ, Narendra KL, Anil KK, Agrawal GP. Development of simultaneous spectrophotometric method of mesalazine and prednisolone in same dosage form. Int J Appl Pharm, 2010; $2: 8-11$.

Moorthi C, Kathiresan K. Curcumine piperine/curcumine quercetin/curcumine silibinin dual drug loaded nanoparticulate combination therapy: a novel approach to target and treat multidrug resistant cancers. J Med Hypotheses Ideas, 2013; 7:15-20. 
Moorthi C, Kathiresan K. Nanotoxicology: toxicity of engineered nanoparticles and approaches to produce safer nanotherapeutics. Int J Pharm Sci, 2012; 2:117-24.

Moorthi C, Kiran K, Manavalan R, Kathiresan K. Preparation and characterization of curcumin-piperine dual drug loaded nanoparticles. Asian Pac J Trop Biomed, 2012; 2:841-8.

Moorthi C, Manavalan R, Kathiresan K. Nanotherapeutics to overcome conventional cancer chemotherapy limitations. J Pharm Pharm Sci, 2011; 14:67-77.

Moorthi C, Senthil K, Mohan S, Kiran K, Kathiresan K. Application of validated RPeHPLCePDA method for the simultaneous estimation of curcumin and piperine in Eudragit E 100 nanoparticles. J Pharm Res, 2013; 7:224-9.

Murti YB, Hartini YS, Hinrichs WLJ, Frijlink HW, Setyaningsih D. UV-Vis spectroscopy to enable determination of the dissolution behavior of solid dispersions containing curcumin as well as piperine. J Young Pharm, 2019; 11:26-30.

Nagappan KV, Meyyanathan SN, Raja RB, Kannan E. A liquid chromatography method for the simultaneous determination of curcumin and piperine in food products using diode array detection. Asian J Res Chem, 2009; 2:115-8.

Nurul H, Halimaton H, Lee SL. Piperine loaded silica aerogel and silica xerogel as NANO-enabled drug delivery system. World Appl Sci J, 2010; 9: 06-16.

Prerana S, Virendra KD, Mohant S, Mishra SK, Rajeev J, Edwards G. Development and validation of a reversed phase HPLC method for simultaneous determination of curcumin and piperine in human plasma for application in clinical pharmacological studies, J Liq Chromatogr Relat Technol, 2009; 32:2961-74.

Shaikh J, Ankola DD, Beniwal V, Singha D, Ravi Kumar MNV. Nanoparticle encapsulation improves oral bioavailability of curcumin by at least 9-fold when compared to curcumin administered with piperine as absorption enhancer. Eur J Pharm Sci, 2009; 37:223-30.
Shanmugam R, Gowthamarajan K, Priyanka DL, Madhuri $\mathrm{K}$, Lokesh M. Elango K. Development and validation of simultaneous estimation method for curcumin and piperine by RP-UFLC. Pak J Pharm Sci, 2014; 27:901-6.

Sharma RA, Gescher AJ, Steward WP. Curcumin: the story so far. Eur J Cancer, 2005; 41:1955-68.

Suchitra P, Rajashree H. A new stability-indicating RP-HPLC method for determination of curcumin: an application to the nanoparticulate formulation. Int J Pharm Pharm Sci, 2016; 8:149-55.

Tushar KK, Darshil BS, Dilip GM. Development and validation of q-absorbance ratio spectrophotometric method for simultaneous estimation of cilnidipine and metoprolol succinate in bulk and combined dosage form. Int J Pharm Pharm Sci, 2014; 6:401-7.

Vipul U, Neeru S, Himanshu MJ, Amreesh M, Manoj M, Singh BP, Sanjeev T. Development and validation of rapid RPHPLC method for estimation of piperine in Piper nigrum L.. Int J Herb Med, 2013; 1:6-9.

Xiu-Mei W, Qi-Zhi Z, Jian Y, Rong-Hua Z, Jun Z, Li-Jing C, Wen-Xing P. Validated HPLC-MS/MS method for simultaneous determination of curcumin and piperine in human plasma. Trop J Pharm Res, 2012; 11:621-9.

\section{How to cite this article:}

Bhairy S, Shaikh A, Nalawade V, Hirlekar R. Development and validation of bivariate UV-visible spectroscopic method for simultaneous estimation of curcumin and piperine in their combined nanoparticulate system. J Appl Pharm Sci, 2021; 11(05):064-070. 